$\square$ Math-Net.Ru

Общероссийский математический портал 
Е. К. Логинов, О вложении аналитических луп Муфанг в альтернативные алгебры, Матем. заметки, 2001, том 69, выпуск 2, 313-315

DOI: https://doi.org/10.4213/mzm683

Использование Общероссийского математического портала Math-Net.Ru подразумевает, что вы прочитали и согласны с пользовательским соглашением 
http://www. mathnet.ru/rus/agreement

Параметры загрузки:

IP: 54.162 .127 .20

26 апреля 2023 г., 16:56:37 


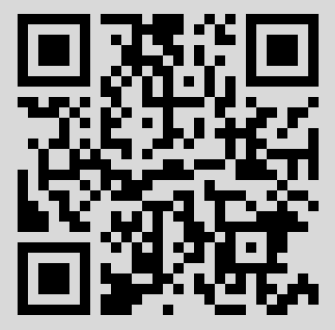




\section{О ВЛОЖЕНИИ АНАЛИТИЧЕСКИХ ЛУП МУФАНГ В АЛЬТЕРНАТИВНЫЕ АЛГЕБРЫ}

\section{Е.К. Логинов}

1. Мальцевым [1] и Кузьминым [2] было установлено соответствие между локальными аналитическими лупами Муфанг и конечномерными алгебрами Мальцева над полем вещественных чисел, аналогичное классическому соответствию Ли между локаљными группами Ли и алгебрами Ли. Позже Кердман [3] показал, что эти результаты полностью переносятся на аналитические лупы Муфанг. А именно, существует единственная с точностью до изоморфизма односвязная аналитическая лупа Муфанг $G$ в целом с данной касательной алгеброй Мальцева и любая связная аналитическая лупа Муфанг $G^{\prime}$ с той же касательной алгеброй может быть получена из $G$ факторизацией по дискретной центраљьной нормальной подлупе, причем пространство односвязной аналитической лупы Муфанг, имеющей разрешимую касательную алгебру Мальцева, гомеоморфно евклидову пространству. В данной заметке устанавливается связь между аналитическими лупами Муфанг и лупами обратимых элементов альтернативных алгебр над полем вещественных чисел.

(C) Е. К. Логинов 
2. Напомним основные понятия теории линейных представлений луп Муфанг (см. [4]). Пусть $G$ - лупа Муфанг. Лупа $G$ выделяется из класса всех луп тождествами

$$
a b \cdot c a=a(b c \cdot a), \quad a(b \cdot c b)=(a b \cdot c) b, \quad(c b \cdot c) a=c(b \cdot c a),
$$

которые назьвают чентральным, правым и левым тождеством Муфанг соответственно. Хорошо известно, что в произвольной лупе два из них являются следствием третьего. Пусть $M-$ линейное пространство над полем $F$ и $\left(\rho_{a}, \lambda_{a}\right)$ - обратимые линейные преобразования $M$, где $a \in G$. Определим на множестве $\widetilde{G}=G \times M$ умножение

$$
(a, x)(b, y)=\left(a b, x \rho_{b}+y \lambda_{a}\right) .
$$

Если отображения $(\rho, \lambda): G \rightarrow$ Aut $M$ таковы, что полученный группоид $\widetilde{G}$ снова является лупой Муфанг, то векторное пространство $M$ называется $G$-модулем, а упорядоченная пара отображений $(\rho, \lambda)$ - линейным представлением лупы $G$. Если $G$ - аналитическая лупа Муфанг, то дополнительно требуется, чтобы и лупа $\widetilde{G}$ была аналитической. Часто удобно использовать краткие обозначения $x a$ и $a x$ вместо введенных выше $x \rho_{a}$ и $x \lambda_{a}$.

Линейное представление $(\rho, \lambda)$ назьвается точным, если ядро этогопредставления $\operatorname{Ker}(\rho, \lambda)=$ $\left\{a \in G \mid \rho_{a}=\lambda_{a}=\mathrm{id}\right\}$ совпадает с единицей лупы $G$. Справедливо

ПредлоЖЕнИЕ 1. Ядро $\operatorname{Ker}(\rho, \lambda)$ линейного представления лупы Муфанг является ее нормальной подлупой.

Пусть $F[G]$ - формальная линейная оболочка лупы Муфанг $G$ и $M$ - некоторый $G$-модуль. Продолжим представление $(\rho, \lambda): G \rightarrow$ Aut $M$ лупы с $G$ на $F[G]$ по $F$-линейности. Получим представление $(\widetilde{\rho}, \widetilde{\lambda}): F[G] \rightarrow$ End $M$ алгебры $F[G]$ в смысле Эйленберга [5]. Связь представлений $(\rho, \lambda)$ и $(\widetilde{\rho}, \tilde{\lambda})$ описьвает

ПреДЛОЖЕнИЕ 2. Пара отображсенй $(\rho, \lambda): G \rightarrow$ Aut $M$ является линейным представлением лупь Муфанг $G$ тогда и только тогда, когда выполняются тождества $x(b \cdot a b)=(x b \cdot a) b,(a b \cdot a) x=a(b \cdot a x)$ и ассочиатор $(a, b, x)$ кососимметричен для всех $a, b \in F[G] u x \in M$.

Заметим, что ядро $\operatorname{Ker}(\widetilde{\rho}, \widetilde{\lambda})=\left\{a \in F[G] \mid \widetilde{\rho}_{a}=\widetilde{\lambda}_{a}=0\right\}$ построенного представления является идеалом в $F[G]$. Причем справедливо

ПРеДЛОЖЕнИЕ 3. Факторалъебра $F[G] / \operatorname{Ker}(\widetilde{\rho}, \widetilde{\lambda})$ альтернативна.

Сформулированные предложения (см. [4]) позволяют доказать следующий критерий вложимости луп Муфанг в лупы обратимых элементов альтернативных алгебр.

Tеорема 1. Лупа Муфанг G вкладьвается в лупу обратимых әлементов альтернативной алгебры тогда и только тогда, когда она обладает точным представлением.

ДоКАЗАТЕЛЬство. Пусть лупа $G$ вложена в лупу обратимых элементов альтернативной алгебры $A$. Выделим в $A$ подалгебру $B$, порожденную элементами лупы $G$. Операторы $R_{a}$ и $L_{a}$ правого и левого умножения элементов $B$ на элемент $a \in G$ обратимы, удовлетворяют условиям предложения 2 и совпадают с тождественньм оператором, только если $a=e$, где $e$ - единица лупы $G$. Поэтому пара отображений $(R, L)$ лупы $G$ в группу Aut $B$ автоморфизмов линейного пространства $B$, сопоставляющих элементу $a \in G$ операторы $R_{a}$ и $L_{a}$, является точным представлением лупы Муфанг $G$.

Обратно, пусть лупа $G$ обладает точньм представлением $(\rho, \lambda): G \rightarrow$ Aut $M$. Продолжим представление $(\rho, \lambda)$ с $G$ на $F[G]$ по $F$-линейности. Получим представление $(\widetilde{\rho}, \widetilde{\lambda}): F[G] \rightarrow$ End $M$ формальной линейной оболочки $F[G]$. Гомоморфизм $\widetilde{\varphi}: F[G] \rightarrow F[G] / \operatorname{Ker}(\widetilde{\rho}, \widetilde{\lambda})$ алгебр индуцирует гомоморфизм $\varphi: G \rightarrow G / H$ луп. Покажем, что подлупа $H$ совпадает с ядром $\operatorname{Ker}(\rho, \lambda)$ представления $(\rho, \lambda)$.

Действительно, если $g \in \operatorname{Ker}(\rho, \lambda)$, то $e-g \in \operatorname{Ker}(\widetilde{\rho}, \widetilde{\lambda})$ и $0=\varphi(e-g)=\varphi(e)-\varphi(g)$. Поэтому $g \in H$ и $\operatorname{Ker}(\rho, \lambda) \subseteq H$. Обратно, если $g \in H$, то $0=\varphi(e)-\varphi(g)=\varphi(e-g)$. Следовательно, $e-g \in \operatorname{Ker}(\widetilde{\rho}, \widetilde{\lambda}), g \in \operatorname{Ker}(\rho, \lambda)$ и $H \subseteq \operatorname{Ker}(\rho, \lambda)$. Таким образом, $H=\operatorname{Ker}(\rho, \lambda)$. Отсюда ввиду точности представления $(\rho, \lambda)$ следует вложение лупы $G$ в лупу обратимых элементов факторалгебры $F[G] / \operatorname{Ker}(\widetilde{\rho}, \widetilde{\lambda})$, которая по предложению 3 альтернативна. Теорема доказана. 
3. Пусть $G$ - аналитическая лупа Муфанг в целом. В окрестности $U$ единицы операция умножения в $G$ выражается через операции сложения и умножения в касательной алгебре Мальцева $M$ с помощью ряда Кемпбелла-Хаусдорфа

$$
a b=x+y+\frac{1}{2}[x, y]+\frac{1}{12}[[x, y], y]+\frac{1}{12}[x,[x, y]]+\cdots .
$$

Определим отображения $(\rho, \lambda): U \rightarrow$ Aut $M$ равенствами

$$
\rho_{b}=\sum_{n=0}^{\infty} \frac{B_{n}}{n !}\left(R_{y}\right)^{n}, \quad \lambda_{a}=\sum_{n=0}^{\infty} \frac{B_{n}}{n !}\left(-R_{x}\right)^{n},
$$

где $R_{y}$ и $R_{x}$ - операторы правого умножения на элементы $y$ и $x$ соответственно, а $B_{n}$ - числа Бернулли. Очевидно, операторы $\rho_{b}$ и $\lambda_{a}$ аналитичны, обратимы и удовлетворяют условиям предложения 2. Поэтому отображения $(\rho, \lambda): U \rightarrow$ Aut $M$ определяют представление локальной лупы $U$ и наделяют множество $\widetilde{U}=U \times M$ структурой локальной аналитической лупы Муфанг. Ясно, что построенное представление является точным.

Пусть $\widetilde{G}$ - аналитическая лупа Муфанг, локально изоморфная лупе $\widetilde{U}$ и содержащая $G$ в качестве подлупы. Поскольку связная лупа алгебраически порождается элементами любой окрестности единицы и для любых элементов $g_{i}, g_{j}$ локальной лупы Муфанг $U$ справедливы тождества

$$
\lambda_{g_{j} g_{i}}=\rho_{g_{i}} \lambda_{g_{i}} \lambda_{g_{j}} \rho_{g_{i}^{-1}}, \quad \rho_{g_{i} g_{j}}=\lambda_{g_{i}} \rho_{g_{i}} \rho_{g_{j}} \lambda_{g_{i}^{-1}},
$$

умножение в $\widetilde{G}$ может быть представлено в виде (1), где элементы $a, b$ лежат в лупе $G$, а операторы $\rho_{b}, \lambda_{a}$ порождаются операторами $\rho_{g_{j}}, \lambda_{g_{i}}$. Таким образом, определено представление $(\rho, \lambda)$ : $G \rightarrow \operatorname{Aut} M$ лупы $G$. Из точности представления локальной лупы $U$ и предложения 1 следует, что ядро $H=\operatorname{Ker}(\rho, \lambda)$ построенного представления - дискретная нормальная подлуппа $G$. Из связности лупы $G$ следует, что $H$ находится в ее центре. Поэтому существует регулярное представление $(\rho, \lambda): G \rightarrow$ Aut $F[H]$, где $\rho_{a}=R_{a}, \lambda_{a}=L_{a}$, если $a \in H$, и $\rho_{a}=\lambda_{a}=$ id, если $a \notin H$. Индуцированное представление $(\rho, \lambda): G \rightarrow \operatorname{Aut}(M \otimes F[H])$, очевидно, является точным. Таким образом, из теоремы 1 следует

Теорема 2. Всякая аналитическая лупа Муфанг вкладывается в лупу обратимых әлементов некоторой альтернативной алгебры над полем вещественных чисел.

\section{СПИСОК ЦИТИРОВАННОЙ ЛИТЕРАТУРЫ}

1. Мальцев А. И. // Матем. сб. 1955. Т. 36. С. 569-576. 2. Кузьмин Е. Н. // Алгебра и логика. 1971. Т. 10. С. 3-22. 3. Кердман Ф. С. // Алгебра и логика. 1979. Т. 18. С. 523-555. 4. Логинов Е. К. // Матем. заметки. 1993. Т. 54. №6. С. 66-73. 5. Eilenberg S. // Ann. Soc. Polon. Mat. 1948. V. 21. P. 125-134. 